\title{
Effect of Molecular Structure on the $\mathrm{CO}_{2}$ Separation Properties of Hydrophobic Solvents Consisting of Grafted Poly Ethylene Glycol and Poly Dimethyl Siloxane Units
}

Robert L. Thompson, ${ }^{1,2,{ }^{*}}$ Jeff Culp, ${ }^{1,2}$ Surya P. Tiwari, ${ }^{1,2}$ Omar Basha, ${ }^{3}$ Wei Shi, ${ }^{1,2}$ Krishnan Damodaran, ${ }^{4}$ Kevin Resnik, ${ }^{1,2}$ Nick Siefert, ${ }^{1}$ and David Hopkinson ${ }^{1}$

1 - U.S. Dept. of Energy, National Energy Technology Laboratory, 626 Cochrans Mill Rd., Pittsburgh, PA 15236 USA

2 - Leidos Research Support Team, National Energy Technology Laboratory, 626 Cochrans Mill Rd., Pittsburgh, PA 15236 USA

3 - Department of Chemical Engineering, University of Pittsburgh, Pittsburgh, PA 15260 USA

4 - Department of Chemistry, Chevron Science Center, University of Pittsburgh, Pittsburgh, PA 15260

USA

* - corresponding author, e-mail address: robert.thompson@netl.doe.gov; tel: 412-386-4953

\section{Synthesis Details}

Structures of the reagents used in this work are depicted in SI Figure 1, while the reactions schemes used to prepare the products reported are shown in SI Figure 2, as reported previously. ${ }^{1}$

Synthesis of linear-3PEG (1): A $500 \mathrm{~mL}$ round bottomed flask was charged with 2,5,8trioxaundec-11-ene (18.7 g, $117 \mathrm{mmol}, 1.09$ equiv.), $0.3 \mathrm{~mL}$ platinum (0)-1,3-divinyl-1,1,3,3tetramethyldisiloxane complex solution ( $2 \% \mathrm{Pt}$ in xylene), and a stir bar. The resultant homogeneous solution was cooled to $20^{\circ} \mathrm{C}$ in a water bath and slowly treated drop-wise with 1,1,1,3,3,5,5-heptamethyltrisiloxane $(23.8 \mathrm{~g}, 107.4 \mathrm{mmol})$. The rate of dropping was controlled to occur over $30 \mathrm{~min}$. time. The temperature was maintained around $20^{\circ} \mathrm{C}$ for 1 hour during which the clear reaction solution eventually became dark yellow. The resultant solution was left to stir at room temperature overnight. This solution was washed with $3 \times 100 \mathrm{~mL} 2 \%$ aqueous $\mathrm{NaCl}$ to extract any unreacted olefin. The organic layer was filtered and evaporated to give a brown liquid (27.8 g, $72.8 \mathrm{mmol}, 68 \%$ isolated yield). This liquid was vacuum distilled to give a clear colorless product $\left(99 \%\right.$ purity by GC). ${ }^{1} \mathrm{H}$ NMR $\left(\mathrm{CDCl}_{3}\right): \delta$ ppm 3.70-3.54 $\left(\mathrm{m}, \mathrm{O}\left(\mathrm{CH}_{2} \mathrm{CH}_{2} \mathrm{O}\right)_{2}, 8 \mathrm{H}\right)$, 3.44$3.37\left(\mathrm{~m}, \mathrm{CH}_{3} \mathrm{O}+\mathrm{OCH}_{2}, 5 \mathrm{H}\right), 1.60$ (br d, $\left.\mathrm{CH}_{2} \mathrm{CH}_{2} \mathrm{CH}_{2}, 2 \mathrm{H}\right), 0.56-0.41\left(\mathrm{~m}, \mathrm{CH}_{2} \mathrm{Si}, 2 \mathrm{H}\right), 0.12-0.00$ (m, $\left.\mathrm{SiCH}_{3}, 19 \mathrm{H}\right) .{ }^{13} \mathrm{C} \mathrm{NMR}\left(\mathrm{CDCl}_{3}\right): \delta \mathrm{ppm} 74.2$ (s, 1C), 71.9 (s, 1C), 70.6 (s, 1C), 70.5 (s, 1C), 70.0 (s, 1C) 59.0 (s, 1C), 23.3 (s, 1C), 14.1 (s, 1C), 1.78-0.07 (s, 7C). ESI MS: calc'd for $\mathrm{C}_{15} \mathrm{H}_{42} \mathrm{NO}_{5} \mathrm{Si}_{3}$ $\left(\mathrm{M}+\mathrm{NH}_{4}\right)^{+}$400.23652, found 400.23582; calc'd for $\mathrm{C}_{15} \mathrm{H}_{38} \mathrm{NaO}_{5} \mathrm{Si}_{3}(\mathrm{M}+\mathrm{Na})^{+} 405.19192$, found 405.19163.

Synthesis of linear-4PEG (2): A method similar to that described for (1) was employed using 2,5,8,11-tetraoxatetradec-13-ene, $\mathrm{Pt}$ catalyst, and 1,1,1,3,3,5,5-heptamethyltrisiloxane which was vacuum distilled to give a clear colorless liquid ( $85 \%$ isolated yield, $96 \%$ purity by GC). ${ }^{1} \mathrm{H}$ $\operatorname{NMR}\left(\mathrm{CDCl}_{3}\right): \delta$ ppm 3.70-3.53 $\left(\mathrm{m}, \mathrm{O}\left(\mathrm{CH}_{2} \mathrm{CH}_{2} \mathrm{O}\right)_{3}, 11 \mathrm{H}\right), 3.44-3.36\left(\mathrm{~m}, \mathrm{CH}_{3} \mathrm{O}+\mathrm{OCH}_{2}, 5 \mathrm{H}\right), 1.68-$ $1.53\left(\mathrm{~m}, \mathrm{CH}_{2} \mathrm{CH}_{2} \mathrm{CH}_{2}, 2 \mathrm{H}\right), 0.51-0.41\left(\mathrm{~m}, \mathrm{CH}_{2} \mathrm{Si}, 2 \mathrm{H}\right), 0.12-0.00\left(\mathrm{~m}, \mathrm{SiCH}_{3}, 21 \mathrm{H}\right)$. ${ }^{13} \mathrm{C} \mathrm{NMR}$ $\left(\mathrm{CDCl}_{3}\right): \delta \mathrm{ppm} 74.2(\mathrm{~s}, 1 \mathrm{C}), 71.9(\mathrm{~s}, 1 \mathrm{C}), 70.6-70.5(\mathrm{~m}, 5 \mathrm{C}), 69.9$ (s, 1C), 58.9 (s, 1C), 23.3 (s, 1C), 14.1 (s, 1C), 1.77 (s, 3C), 1.22 (s, 2C), 0.07 (s, 2C). ESI MS: calc'd for $\mathrm{C}_{17} \mathrm{H}_{46} \mathrm{NO}_{6} \mathrm{Si}_{3}$ $\left(\mathrm{M}+\mathrm{NH}_{4}\right)^{+}$444.26250, found 444.26274; calc'd for $\mathrm{C}_{17} \mathrm{H}_{42} \mathrm{NaO}_{6} \mathrm{Si}_{3}(\mathrm{M}+\mathrm{Na})^{+} 449.21811$, found 449.21814 .

Synthesis of linear-5PEG (3): A method similar to that described for (1) was employed using 2,5,8,11,14-pentaoxaheptadec-16-ene, Pt catalyst, and 1,1,1,3,5,5,5-heptamethyl-trisiloxane 
which was vacuum distilled to give a clear colorless liquid (43\% isolated yield, $87 \%$ purity by GC). ${ }_{1}^{1} \mathrm{H}$ NMR $\left(\mathrm{CDCl}_{3}\right): \delta \mathrm{ppm} 3.68-3.53\left(\mathrm{~m}, \mathrm{O}\left(\mathrm{CH}_{2} \mathrm{CH}_{2} \mathrm{O}\right)_{4}, 16 \mathrm{H}\right), 3.43-3.36\left(\mathrm{~m}, \mathrm{CH}_{3} \mathrm{O}+\mathrm{OCH} \mathrm{CH}_{2}, 5 \mathrm{H}\right)$, 1.68-1.53 (m, $\left.\mathrm{CH}_{2} \mathrm{CH}_{2} \mathrm{CH}_{2}, 2 \mathrm{H}\right), 0.56-0.40\left(\mathrm{~m}, \mathrm{CH}_{2} \mathrm{Si}, 2 \mathrm{H}\right), 0.11-0.00\left(\mathrm{~m}, \mathrm{SiCH}_{3}, 22 \mathrm{H}\right)$. ${ }^{13} \mathrm{C} \mathrm{NMR}$ $\left(\mathrm{CDCl}_{3}\right): \delta \mathrm{ppm} 74.0(\mathrm{~s}, 1 \mathrm{C}), 71.8(\mathrm{~s}, 1 \mathrm{C}), 70.5-70.4(\mathrm{~m}, 5 \mathrm{C}), 69.8(\mathrm{~s}, 1 \mathrm{C}), 58.8(\mathrm{~s}, 1 \mathrm{C}), 23.2(\mathrm{~s}$, 1C), 13.9 (s, 1C), $1.62(\mathrm{~s}, 3 \mathrm{C}), 1.06$ (s, 2C), -0.08 (s, 2C). ESI MS: calc'd for $\mathrm{C}_{19} \mathrm{H}_{50} \mathrm{NO}_{7} \mathrm{Si}_{3}$ $\left(\mathrm{M}+\mathrm{NH}_{4}\right)^{+}$470.2881, found 488.2890; calc'd for $\mathrm{C}_{19} \mathrm{H}_{46} \mathrm{NaO}_{7} \mathrm{Si}_{3}(\mathrm{M}+\mathrm{Na})^{+}$493.24426, found 493.24435 .

Synthesis of T-3PEG (4): $:^{1}$ A method similar to that described for (1) was employed using 2,5,8trioxaundec-11-ene, Pt catalyst, and 1,1,3,3,5,5,5-heptamethyltrisiloxane which was vacuum distilled to give a clear colorless liquid (44\% isolated yield, $98 \%$ purity by $\mathrm{GC})$. ${ }^{1} \mathrm{H} \mathrm{NMR}\left(\mathrm{CDCl}_{3}\right)$ : $\delta$ ppm 3.69-3.54 (m, O $\left.\left(\mathrm{CH}_{2} \mathrm{CH}_{2} \mathrm{O}\right)_{2}, 8 \mathrm{H}\right), 3.43-3.36\left(\mathrm{~m}, \mathrm{CH}_{3} \mathrm{O}+\mathrm{OCH}_{2}, 5 \mathrm{H}\right), 1.60$ (br dd, $\left.\mathrm{CH}_{2} \mathrm{CH}_{2} \mathrm{CH}_{2}, 2 \mathrm{H}\right), 0.48-0.39\left(\mathrm{~m}, \mathrm{CH}_{2} \mathrm{Si}, 2 \mathrm{H}\right), 0.08$ (s, $\left.\mathrm{SiCH}_{3}, 18 \mathrm{H}\right), 0.00$ (s, $\left.\mathrm{SiCH}_{3}, 3 \mathrm{C}\right) .{ }^{13} \mathrm{C} \mathrm{NMR}$ $\left(\mathrm{CDCl}_{3}\right): \delta \mathrm{ppm} 74.1$ (s, 1C), 71.9 (s, 1C), 70.6 (s, 1C), 70.5 (s, 1C), 69.9 (s, 1C), 59.0 (s, 1C), 23.1 (s, 1C), 13.5 (s, 1C), $1.8(\mathrm{~s}, 6 \mathrm{C}),-0.4$ (s, 1C). ESI MS: calc'd for $\mathrm{C}_{15} \mathrm{H}_{42} \mathrm{NO}_{5} \mathrm{Si}_{3}\left(\mathrm{M}+\mathrm{NH}_{4}\right)^{+}$ 400.23652, found 400.23617; calc'd for $\mathrm{C}_{15} \mathrm{H}_{38} \mathrm{NaO}_{5} \mathrm{Si}_{3}(\mathrm{M}+\mathrm{Na})^{+}$405.19192, found 405.19204.

Synthesis of T-4PEG (5): A method similar to that described for (1) was employed using 2,5,8,11tetraoxatetradec-13-ene, Pt catalyst, and 1,1,3,3,5,5,5-heptamethyltrisiloxane which was vacuum distilled to give a clear colorless liquid $(78 \%$ isolated yield, $92 \%$ purity by $\mathrm{GC})$. ${ }^{1} \mathrm{H} \mathrm{NMR}\left(\mathrm{CDCl}_{3}\right)$ : $\delta$ ppm 3.73-3.53 $\left(\mathrm{m}, \mathrm{O}\left(\mathrm{CH}_{2} \mathrm{CH}_{2} \mathrm{O}\right)_{3}, 12 \mathrm{H}\right), 3.43-3.37\left(\mathrm{~m}, \mathrm{CH}_{3} \mathrm{O}+\mathrm{OCH}_{2}, 5 \mathrm{H}\right), 1.60$ (br dd, $\left.\mathrm{CH}_{2} \mathrm{CH}_{2} \mathrm{CH}_{2}, 2 \mathrm{H}\right), 0.47-0.40\left(\mathrm{~m}, \mathrm{CH}_{2} \mathrm{Si}, 2 \mathrm{H}\right), 0.08$ (s, $\left.\mathrm{SiCH}_{3}, 18 \mathrm{H}\right), 0.01\left(\mathrm{~s}, \mathrm{SiCH}_{3}, 3 \mathrm{H}\right)$. ${ }^{13} \mathrm{C} \mathrm{NMR}$ $\left(\mathrm{CDCl}_{3}\right): \delta \mathrm{ppm} 74.1$ (s, 1C), $71.9(\mathrm{~s}, 1 \mathrm{C}), 70.6(\mathrm{~m}, 4 \mathrm{C}), 69.9(\mathrm{~s}, 1 \mathrm{C}), 59.0(\mathrm{~s}, 1 \mathrm{C}), 23.2(\mathrm{~s}, 1 \mathrm{C})$, 13.5 (s, 1C), 1.8 (s, 6C), -0.4 (s, 1C). ESI MS: calc'd for $\mathrm{C}_{17} \mathrm{H}_{46} \mathrm{NO}_{6} \mathrm{Si}_{3}\left(\mathrm{M}+\mathrm{NH}_{4}\right)^{+} 444.26274$, found 444.26250; calc'd for $\mathrm{C}_{17} \mathrm{H}_{42} \mathrm{NaO}_{5} \mathrm{Si}_{3}(\mathrm{M}+\mathrm{Na})^{+}$449.21814, found 449.21811.

Synthesis of T-5PEG (6): A method similar to that described for (1) was employed using 2,5,8,11,14-pentaoxaheptadec-16-ene, Pt catalyst, and 1,1,3,3,5,5,5-heptamethyl-trisiloxane which was vacuum distilled to give a clear colorless liquid (65\% isolated yield, $86 \%$ purity by GC). ${ }_{1}^{1} \mathrm{H}$ NMR $\left(\mathrm{CDCl}_{3}\right): \delta$ ppm 3.69-3.53 $\left(\mathrm{m}, \mathrm{O}\left(\mathrm{CH}_{2} \mathrm{CH}_{2} \mathrm{O}\right)_{4}, 16 \mathrm{H}\right), 3.42-3.36\left(\mathrm{~m}, \mathrm{CH}_{3} \mathrm{O}+\mathrm{OCH}_{2}, 5 \mathrm{H}\right)$, 1.59 (br dd, $\mathrm{CH}_{2} \mathrm{CH}_{2} \mathrm{CH}_{2}, 2 \mathrm{H}$ ), 0.47-0.39 (m, $\left.\mathrm{CH}_{2} \mathrm{Si}, 2 \mathrm{H}\right), 0.08$ (s, $\left.\mathrm{SiCH}_{3}, 18 \mathrm{H}\right), 0.00$ (s, $\mathrm{SiCH}_{3}$, 3H). ${ }^{13} \mathrm{C} \mathrm{NMR}\left(\mathrm{CDCl}_{3}\right): \delta \mathrm{ppm} 74.1(\mathrm{~s}, 1 \mathrm{C}), 71.9(\mathrm{~s}, 1 \mathrm{C}), 70.6(\mathrm{~m}, 8 \mathrm{C}), 69.9(\mathrm{~s}, 1 \mathrm{C}), 59.0(\mathrm{~s}, 1 \mathrm{C})$, 23.1 (s, 1C), 13.5 (s, 1C), $1.8(\mathrm{~s}, 6 \mathrm{C}),-0.4(\mathrm{~s}, 1 \mathrm{C})$. ESI MS: calc'd for $\mathrm{C}_{19} \mathrm{H}_{50} \mathrm{NO}_{7} \mathrm{Si}_{3}\left(\mathrm{M}+\mathrm{NH}_{4}\right)^{+}$ 488.28895, found 488.28834; calc'd for $\mathrm{C}_{19} \mathrm{H}_{46} \mathrm{NaO}_{7} \mathrm{Si}_{3}(\mathrm{M}+\mathrm{Na})^{+}$493.24435, found 493.24429.

Synthesis of disub-3PEG (7): A $500 \mathrm{~mL}$ round bottomed flask was charged with 2,5,8trioxaundec-11-ene (64.1 g, $400 \mathrm{mmol}, 2.03$ equiv.), $0.75 \mathrm{~mL}$ platinum (0)-1,3-divinyl-1,1,3,3tetramethyldisiloxane complex solution (2\% $\mathrm{Pt}$ in xylene), and a stir bar. The resultant homogeneous solution was cooled to $20^{\circ} \mathrm{C}$ in a water bath and slowly treated drop-wise with 1,1,3,3,5,5-hexamethyltrisiloxane $(41.1 \mathrm{~g}, 197 \mathrm{mmol}, 1$ equiv). The rate of dropping was controlled to occur over $30 \mathrm{~min}$. time. The temperature was maintained around $20^{\circ} \mathrm{C}$ for 1 hour during which the clear reaction solution eventually became dark yellow. The resultant solution was left to stir at room temperature overnight. This solution was washed with $3 \times 100 \mathrm{~mL} 2 \%$ aqueous $\mathrm{NaCl}$ to extract any unreacted olefin. The organic layer was filtered and evaporated to give a brown liquid, which was passed through a short column of silica gel for purification. The product was then dried at $50^{\circ} \mathrm{C}$ for 3 days. Upon drying, (7) was obtained as a clear pale-brownish liquid $(85.7 \mathrm{~g}, 162 \mathrm{mmol}, 82 \%$ isolated yield, $74 \%$ purity by $\mathrm{GC}) .{ }^{1} \mathrm{H} \mathrm{NMR}\left(\mathrm{CDCl}_{3}\right)$ : $\delta \mathrm{ppm} \mathrm{3.75-}$ $3.53\left(\mathrm{~m}, \mathrm{O}\left(\mathrm{CH}_{2} \mathrm{CH}_{2} \mathrm{O}\right)_{2}, 18 \mathrm{H}\right), 3.43-3.36\left(\mathrm{~m}, \mathrm{CH}_{3} \mathrm{O}+\mathrm{OCH}_{2}, 10 \mathrm{H}\right), 1.59\left(\mathrm{br} \mathrm{d}, \mathrm{CH}_{2} \mathrm{CH}_{2} \mathrm{CH}_{2}, 4 \mathrm{H}\right)$, 
0.59-0.40 (m, $\left.\mathrm{CH}_{2} \mathrm{Si}, 4 \mathrm{H}\right), 0.16-0.00\left(\mathrm{~m}, \mathrm{SiCH}_{3}, 19 \mathrm{H}\right) .{ }^{13} \mathrm{C} \mathrm{NMR}\left(\mathrm{CDCl}_{3}\right): \delta \mathrm{ppm} 74.2(\mathrm{~s}, 2 \mathrm{C})$, 71.9 (s, 2C), 70.6-70.5 (m, 4C), 69.9 (s, 2C), 59.0 (s, 2C), $23.3(\mathrm{~m}, 2 \mathrm{C}), 14.1$ (m, 2C), 1.2-1.1 (m, $2 \mathrm{C}), 0.2-0.1(\mathrm{~m}, 4 \mathrm{C})$. ESI MS: calc'd for $\mathrm{C}_{22} \mathrm{H}_{56} \mathrm{NO}_{8} \mathrm{Si}_{3}\left(\mathrm{M}+\mathrm{NH}_{4}\right)^{+}$546.33082, found 546.33001; calc'd for $\mathrm{C}_{19} \mathrm{H}_{46} \mathrm{NaO}_{7} \mathrm{Si}_{3}(\mathrm{M}+\mathrm{Na})^{+} 551.28622$, found 551.28601.

Synthesis of disub-4PEG (8): A method similar to that described for (7) was employed using 2 equivalents of 2,5,8,11-tetraoxatetradec-13-ene, $\mathrm{Pt}$ catalyst, and 1,1,3,3,5,5hexamethyltrisiloxane (84\% isolated yield, $86 \%$ purity by $\mathrm{GC}) .{ }^{1} \mathrm{H}$ NMR $\left(\mathrm{CDCl}_{3}\right)$ : $\delta \mathrm{ppm} \mathrm{3.67-}$ $3.50\left(\mathrm{~m}, \mathrm{O}\left(\mathrm{CH}_{2} \mathrm{CH}_{2} \mathrm{O}\right)_{3}, 24 \mathrm{H}\right), 3.40-3.33\left(\mathrm{~m}, \mathrm{CH}_{3} \mathrm{O}+\mathrm{OCH}_{2}, 10 \mathrm{H}\right), 1.63-1.51\left(\mathrm{~m}, \mathrm{CH}_{2} \mathrm{CH}_{2} \mathrm{CH}_{2}, 4 \mathrm{H}\right)$,

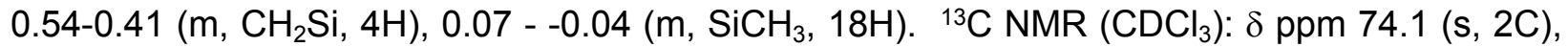
71.8 (s, 2C), $70.5(\mathrm{~m}, 8 \mathrm{C}), 69.9(\mathrm{~s}, 2 \mathrm{C}), 58.9(\mathrm{~s}, 2 \mathrm{C}), 23.3(\mathrm{~m}, 2 \mathrm{C}), 14.0(\mathrm{~m}, 2 \mathrm{C}), 1.17(\mathrm{~m}, 2 \mathrm{C})$, $0.1(\mathrm{~m}, 4 \mathrm{C})$. ESI MS: calc'd for $\mathrm{C}_{26} \mathrm{H}_{64} \mathrm{NO}_{10} \mathrm{Si}_{3}\left(\mathrm{M}+\mathrm{NH}_{4}\right)^{+}$634.38325, found 634.38293; calc'd for $\mathrm{C}_{26} \mathrm{H}_{60} \mathrm{NaO}_{10} \mathrm{Si}_{3}(\mathrm{M}+\mathrm{Na})^{+}$639.33865, found 639.33873.

Synthesis of disub-5PEG (9): A method similar to that described for (7) was employed using 2 equivalents of 2,5,8,11,14-pentaoxaheptadec-16-ene, $\mathrm{Pt}$ catalyst, and 1,1,3,3,5,5hexamethyltrisiloxane (82\% isolated yield, $66 \%$ purity by $\mathrm{GC}) .{ }^{1} \mathrm{H}$ NMR $\left(\mathrm{CDCl}_{3}\right)$ : $\delta \mathrm{ppm} 3.69-$ $3.52\left(\mathrm{~m}, \mathrm{O}\left(\mathrm{CH}_{2} \mathrm{CH}_{2} \mathrm{O}\right)_{4}, 32 \mathrm{H}\right), 3.42-3.35\left(\mathrm{~m}, \mathrm{CH}_{3} \mathrm{O}+\mathrm{OCH}_{2}, 10 \mathrm{H}\right), 1.58\left(\mathrm{br} \mathrm{d}, \mathrm{CH}_{2} \mathrm{CH}_{2} \mathrm{CH}_{2}, 4 \mathrm{H}\right)$, $0.49\left(\mathrm{~m}, \mathrm{CH}_{2} \mathrm{Si}, 4 \mathrm{H}\right), 0.16--0.01\left(\mathrm{~m}, \mathrm{SiCH}_{3}, 19 \mathrm{H}\right) .{ }^{13} \mathrm{C} \mathrm{NMR}\left(\mathrm{CDCl}_{3}\right): \delta \mathrm{ppm} 74.2(\mathrm{~s}, 2 \mathrm{C}), 71.9$ (s, 2C), 70.5-70.4 (m, 12C), $69.9(\mathrm{~s}, 2 \mathrm{C}), 59.0$ (s, 2C), $23.3(\mathrm{~m}, 2 \mathrm{C}), 14.1(\mathrm{~m}, 2 \mathrm{C}), 1.2-1.1(\mathrm{~m}, 2 \mathrm{C})$, 0.6-0.5 (s, 4C). ESI MS: calc'd for $\mathrm{C}_{30} \mathrm{H}_{72} \mathrm{NO}_{12} \mathrm{Si}_{3}\left(\mathrm{M}+\mathrm{NH}_{4}\right)^{+} 722.43568$, found 722.43532; calc'd for $\mathrm{C}_{30} \mathrm{H}_{68} \mathrm{NaO}_{12} \mathrm{Si}_{3}(\mathrm{M}+\mathrm{Na})^{+}$727.39108, found 727.39071 .

The FTIR spectra for all three siloxane reagents show a strong band around $2125 \mathrm{~cm}^{-1}$ assigned to the Si-H bond, while the products formed all lack this bond. FTIR spectra for 1,1,3,3,5,5hexamethyltrisiloxane prior to reaction and the purified disub-4PEG (8) are both shown in SI Figure 3 , in which the $\mathrm{Si}-\mathrm{H}$ band has completely disappeared in the product, indicating that no unreacted siloxane remains.

\section{Figure Captions}

SI Figure 1. Reagents used in the syntheses of hybrid PEG-PDMS solvents: (a) siloxy silanes and (b) PEG-olefins.

SI Figure 2. Reaction schemes employed for preparation of (a) linear, (b) T-shaped, and (c) disubstituted solvents.

SI Figure 3. FTIR spectra of 1,1,3,3,5,5-hexamethyltrisiloxane and disub-4PEG (8).

SI Figure 4. Calculated atomic charges (e-) for all non-hydrogen atoms in solvent molecules of linear-3PEG (1).

SI Figure 5. Calculated atomic charges (e-) for all atoms in solvent molecules of linear-4PEG (2).

SI Figure 6. Calculated atomic charges (e-) for all atoms in solvent molecules of linear-5PEG (3).

SI Figure 7. Calculated atomic charges (e-) for all atoms in solvent molecules of T-3PEG (4).

SI Figure 8. Calculated atomic charges (e-) for all atoms in solvent molecules of T-4PEG (5).

SI Figure 9. Calculated atomic charges (e-) for all atoms in solvent molecules of T-5PEG (6). 
SI Figure 10. Calculated atomic charges (e-) for all atoms in solvent molecules of disub-3PEG (7).

SI Figure 11. Calculated atomic charges (e) for all atoms in solvent molecules of disub-4PEG (8).

SI Figure 12. Calculated atomic charges (e-) for all atoms in solvent molecules of disub-5PEG (9).

SI Figure 13. Calculated interaction distances $(\AA)$ with $\mathrm{CO}_{2}$ and interaction energies $(\mathrm{kJ} / \mathrm{mol})$ for selected PEG oxygen atoms for linear-3PEG (1).

SI Figure 14. Calculated interaction distances $(\AA)$ with $\mathrm{CO}_{2}$ and interaction energies $(\mathrm{kJ} / \mathrm{mol})$ for selected PEG oxygen atoms for linear-4PEG (2).

SI Figure 15. Calculated interaction distances $(\AA)$ with $\mathrm{CO}_{2}$ and interaction energies $(\mathrm{kJ} / \mathrm{mol})$ for selected PEG oxygen atoms for T-3PEG (4).

SI Figure 16. Calculated interaction distances $(\AA)$ with $\mathrm{CO}_{2}$ and interaction energies $(\mathrm{kJ} / \mathrm{mol})$ for selected PEG oxygen atoms for T-4PEG (5).

SI Figure 17. Calculated interaction distances $(\AA)$ with $\mathrm{CO}_{2}$ and interaction energies $(\mathrm{kJ} / \mathrm{mol})$ for selected PEG oxygen atoms for T-5PEG (6).

Table 1: Foaming Experimental Data Point Details

\begin{tabular}{|c|c|c|c|c|c|c|c|}
\hline$\#$ & Liquid & Gas & $\mathrm{T}\left({ }^{\circ} \mathrm{C}\right)$ & $\mathrm{P}(\mathrm{bar})$ & $\mathrm{Q}\left(\mathrm{m}^{3} / \mathrm{s}\right)$ & $\mathrm{H}(\mathrm{cm})$ & $\mathrm{t}(\mathrm{s})$ \\
\hline 1 & disub 4-PEG & $\mathrm{CO}_{2}$ & 20 & 5 & 0.002391 & 1.33 & 112 \\
\hline 2 & disub 3-PEG & $\mathrm{CO}_{2}$ & 20 & 5 & 0.002391 & 1.00 & 63 \\
\hline 3 & T-4PEG & $\mathrm{CO}_{2}$ & 20 & 5 & 0.002391 & 15.95 & 57 \\
\hline & & & & & & & \\
\hline 4 & disub 4-PEG & $\mathrm{CO}_{2}$ & 40 & 5 & 0.002520 & 0.97 & 139 \\
\hline 5 & disub 3-PEG & $\mathrm{CO}_{2}$ & 40 & 5 & 0.002520 & 0.35 & 44 \\
\hline 6 & T-4PEG & $\mathrm{CO}_{2}$ & 40 & 5 & 0.002520 & 10.85 & 65 \\
\hline
\end{tabular}

\section{References}

1. Siefert, N. S.; Agarwal, S.; Shi, F.; Shi, W.; Roth, E. A.; Hopkinson, D.; Kusuma, V. A.; Thompson, R. L.; Luebke, D. R.; Nulwala, H. B., Hydrophobic physical solvents for pre-combustion $\mathrm{CO} 2$ capture: Experiments, computational simulations, and techno-economic analysis. International Journal of Greenhouse Gas Control 2016, 49, 364-371. 


\section{SI Figure 1}

a. siloxysilanes

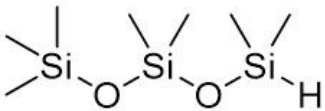

1,1,1,3,3,5,5-heptamethyltrisiloxane

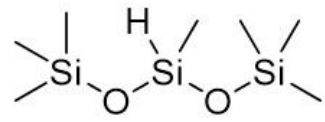

1,1,3,3,5,5,5-heptamethyltrisiloxane

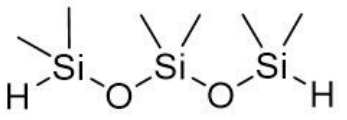

1,1,3,3,5,5-hexamethyltrisiloxane b. PEG-olefins<smiles>C=CCOCCOCCOC</smiles>

2,5,8-trioxaundec-10-ene

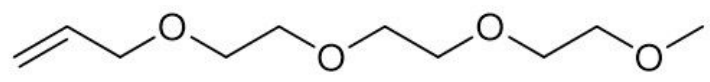

2,5,8,11-tetraoxatetradec-13-ene

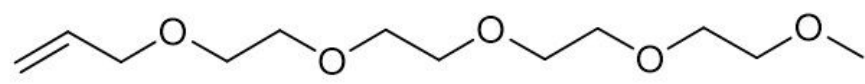

2,5,8,11,13-pentaoxaheptadec-16-ene 


\section{SI Figure 2}

a. linear- $n$ PEG

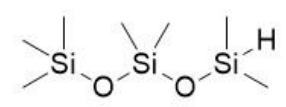

$+$
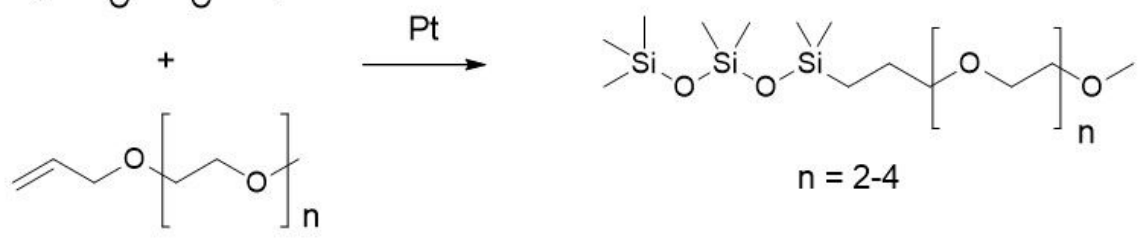

$n=2-4$

b. T-nPEG
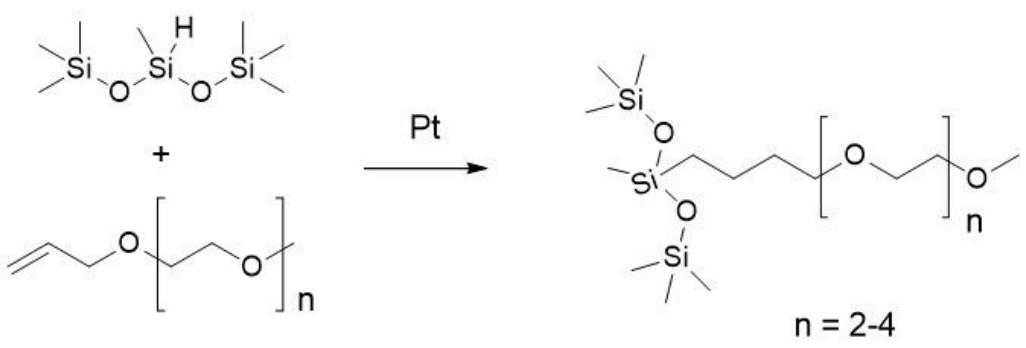

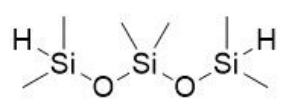

c. disub-nPEG

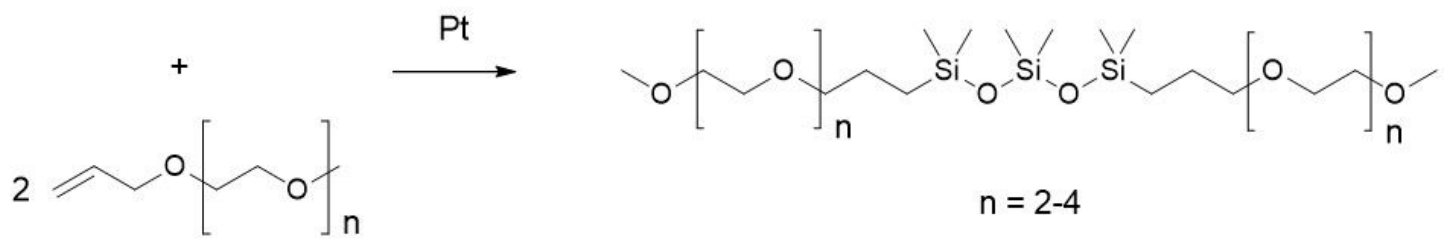


SI Figure 3

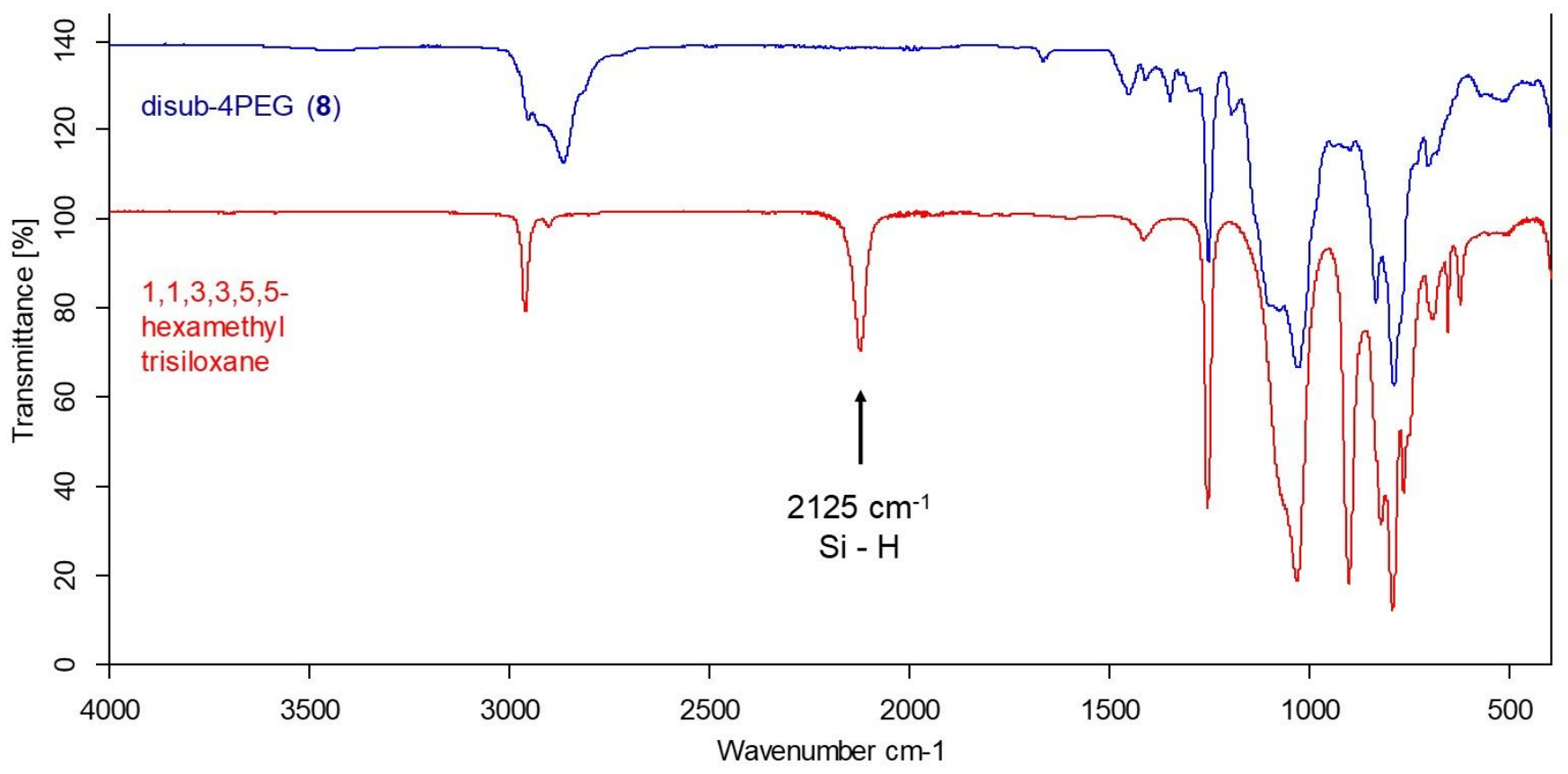




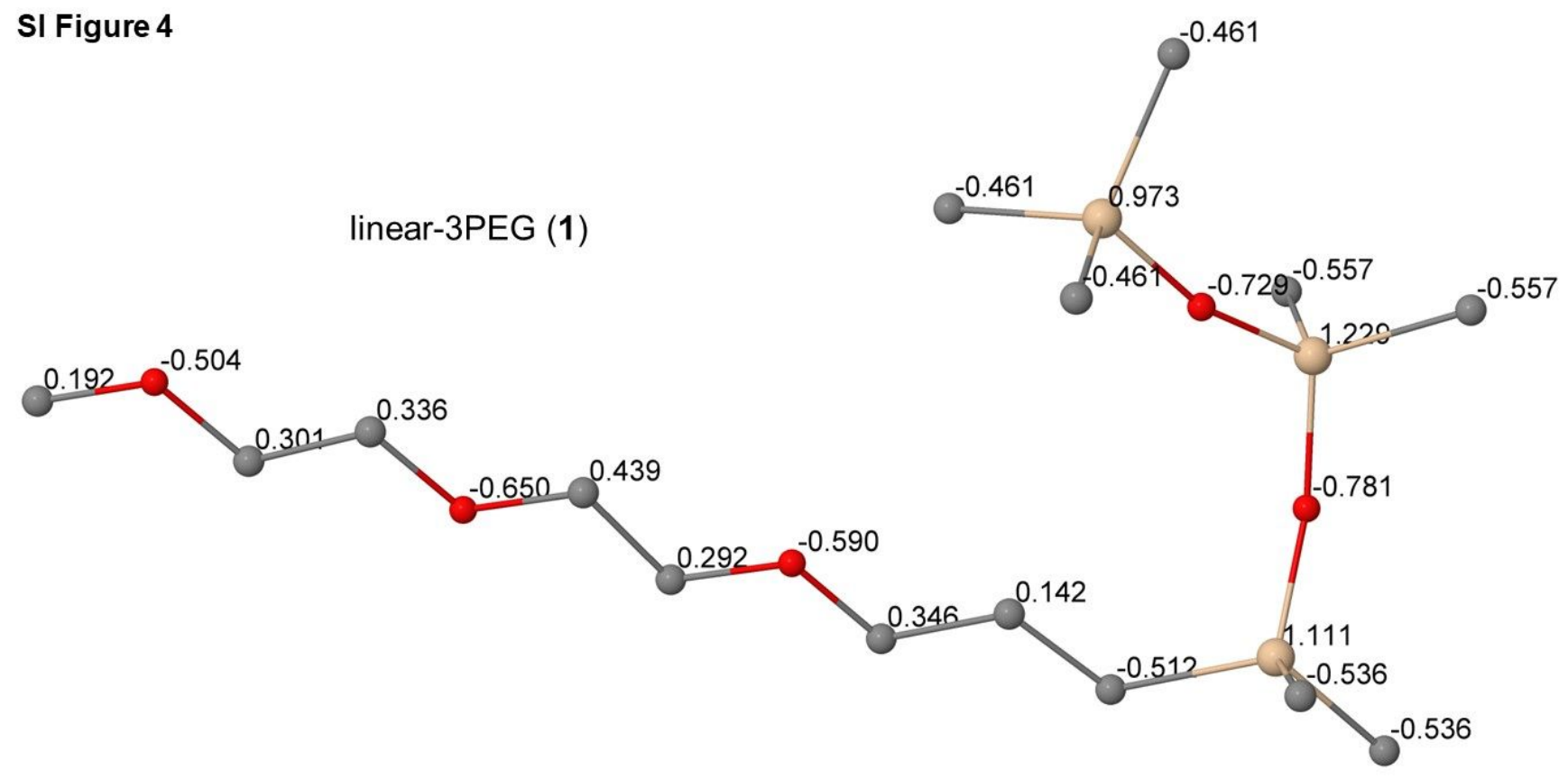

SI Figure 5

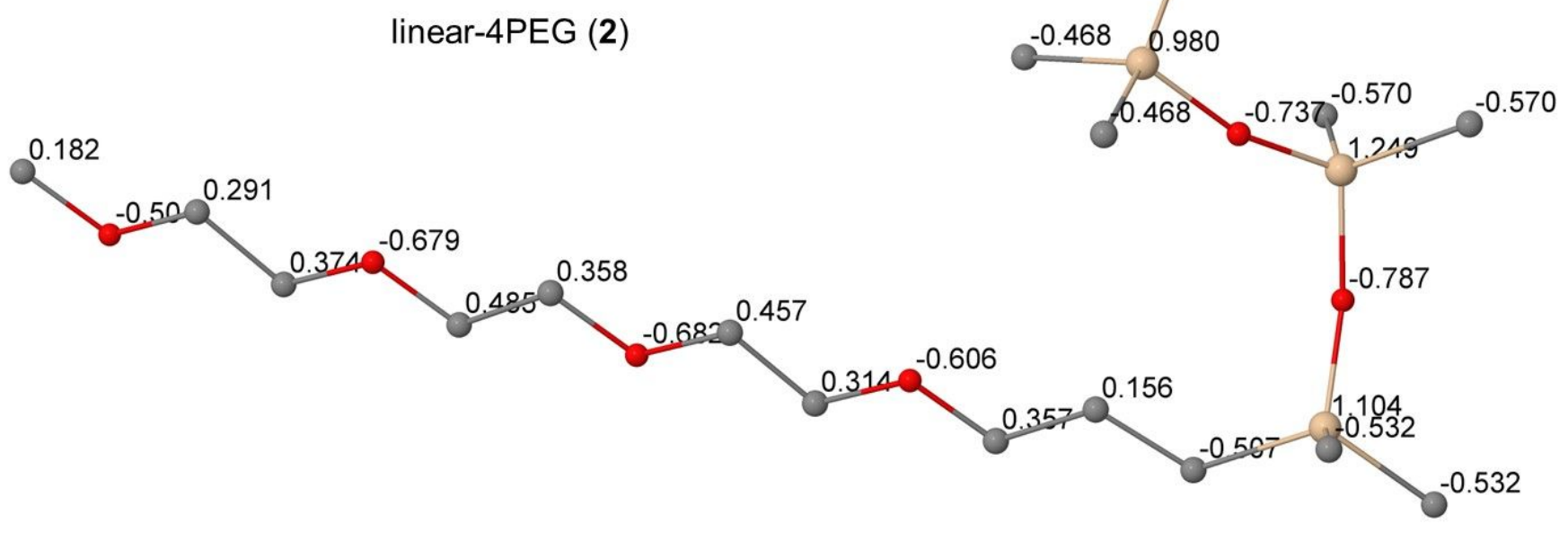



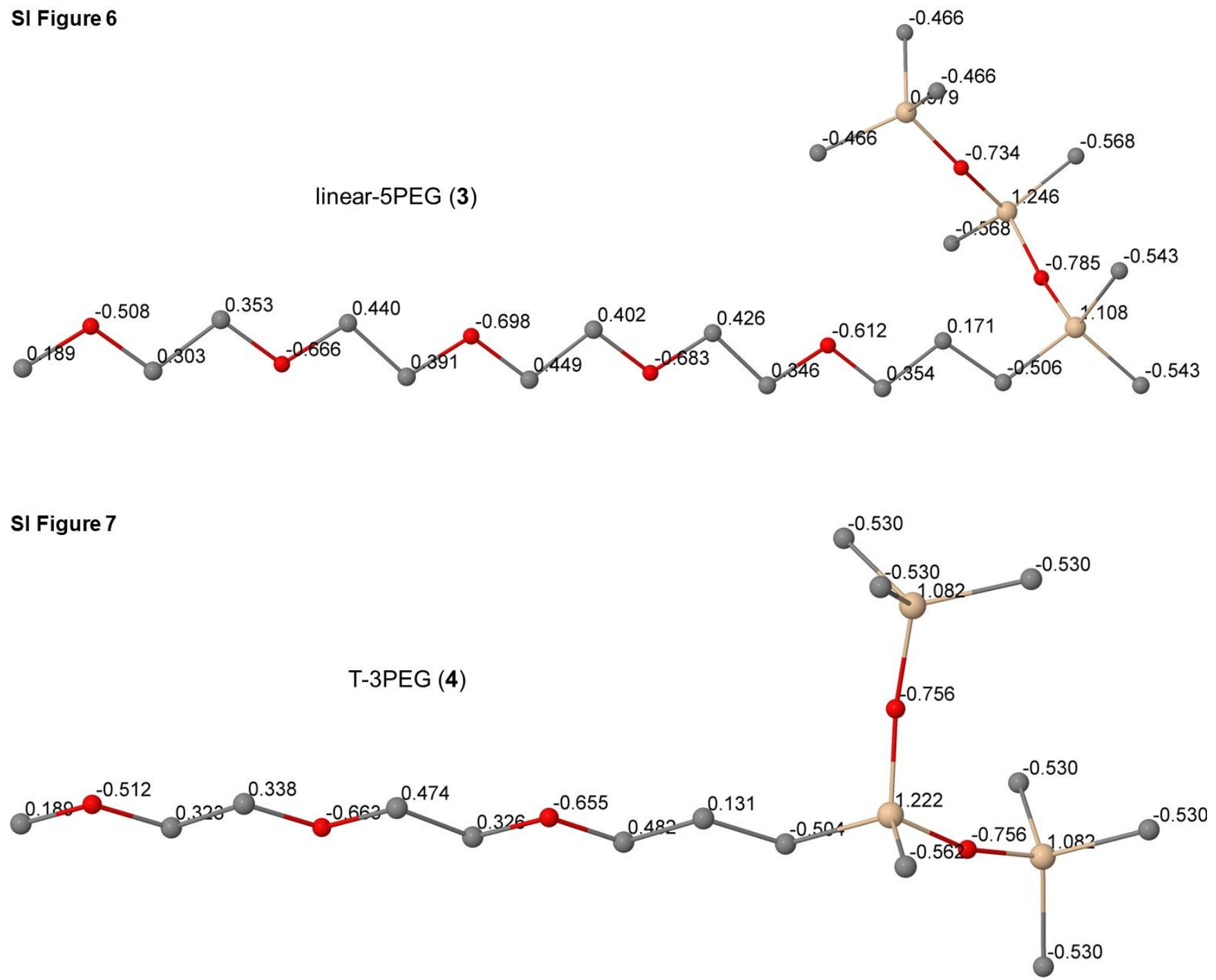

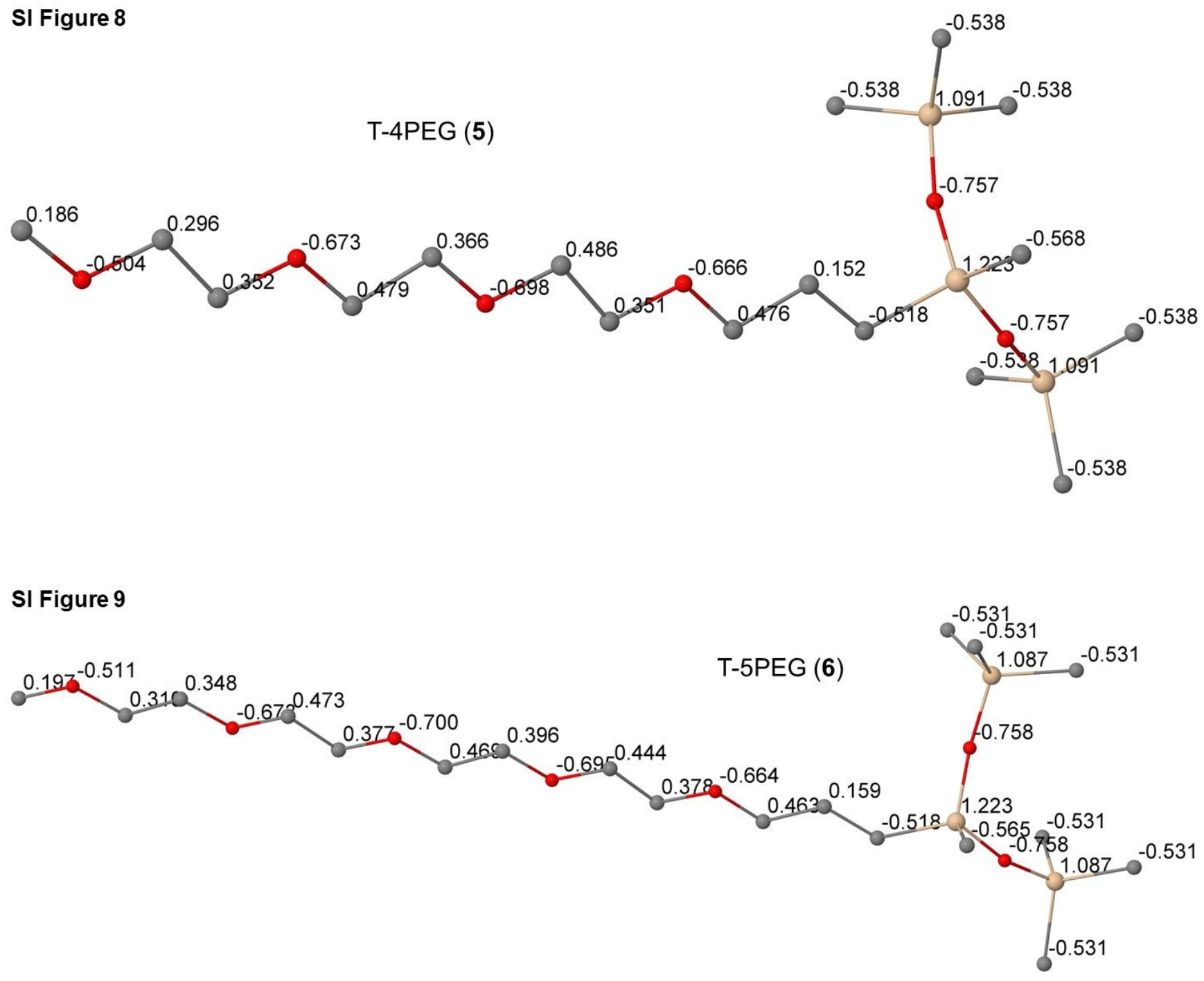

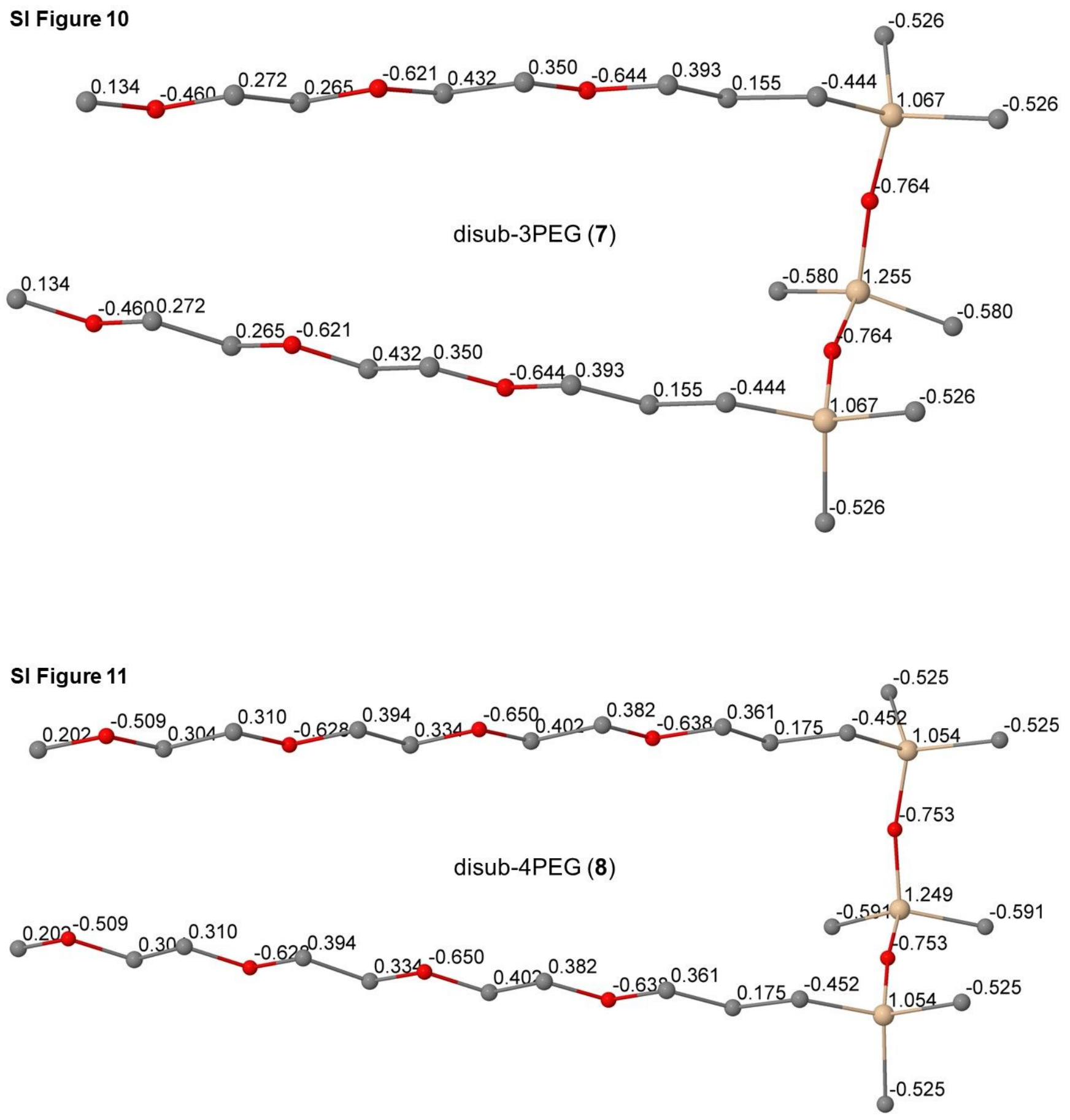


\section{SI Figure 12}

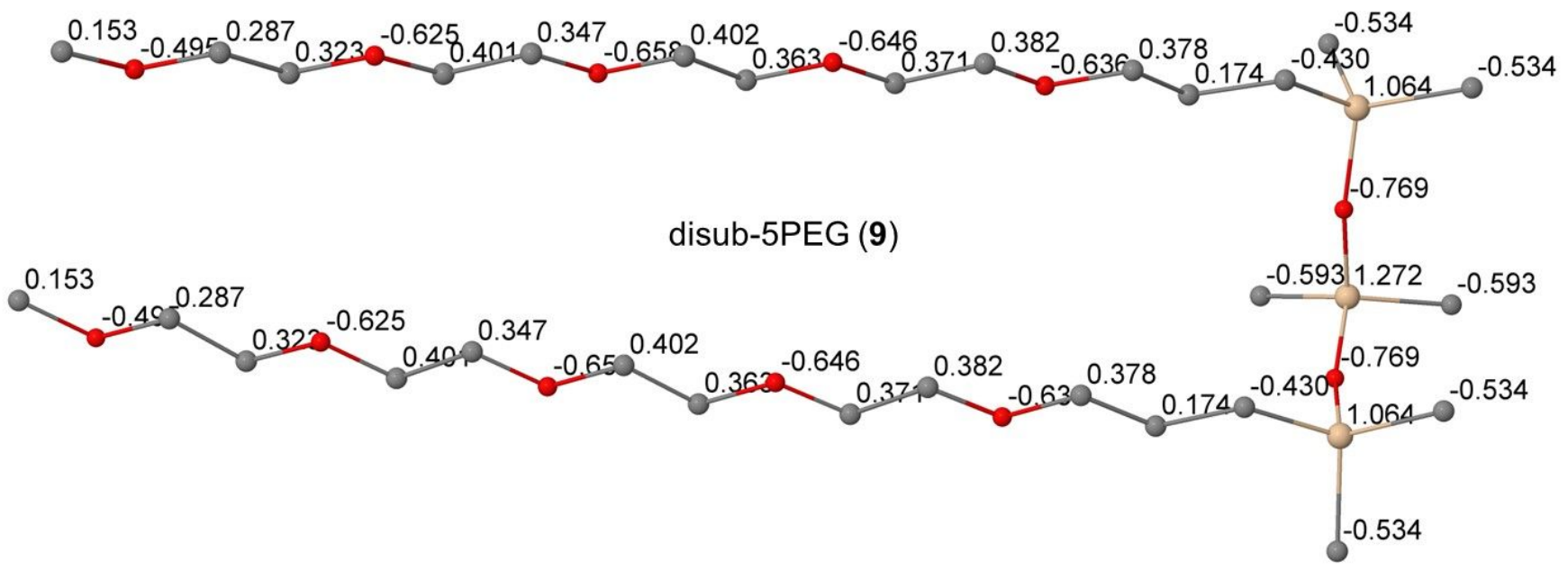

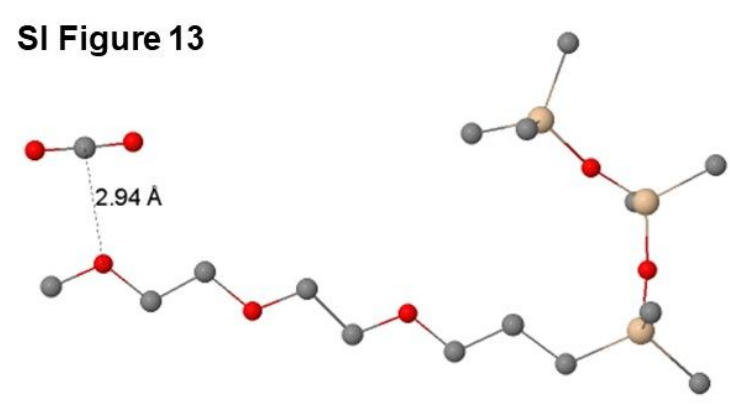

$-12.8565$

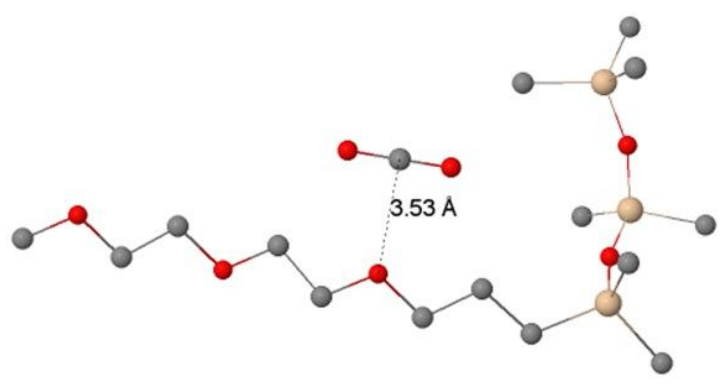

$-10.7816$

linear-3PEG (1)

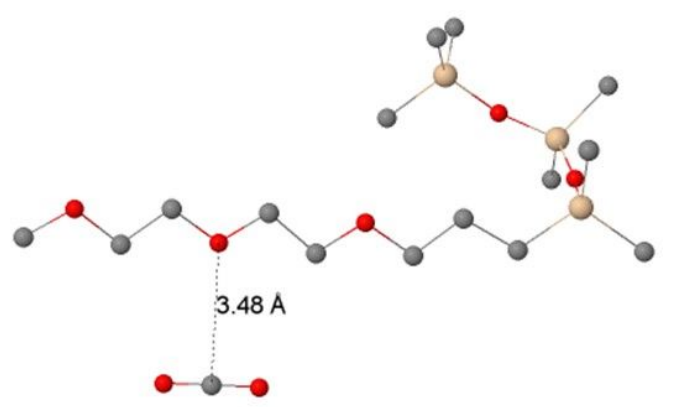

$-9.3135$ 


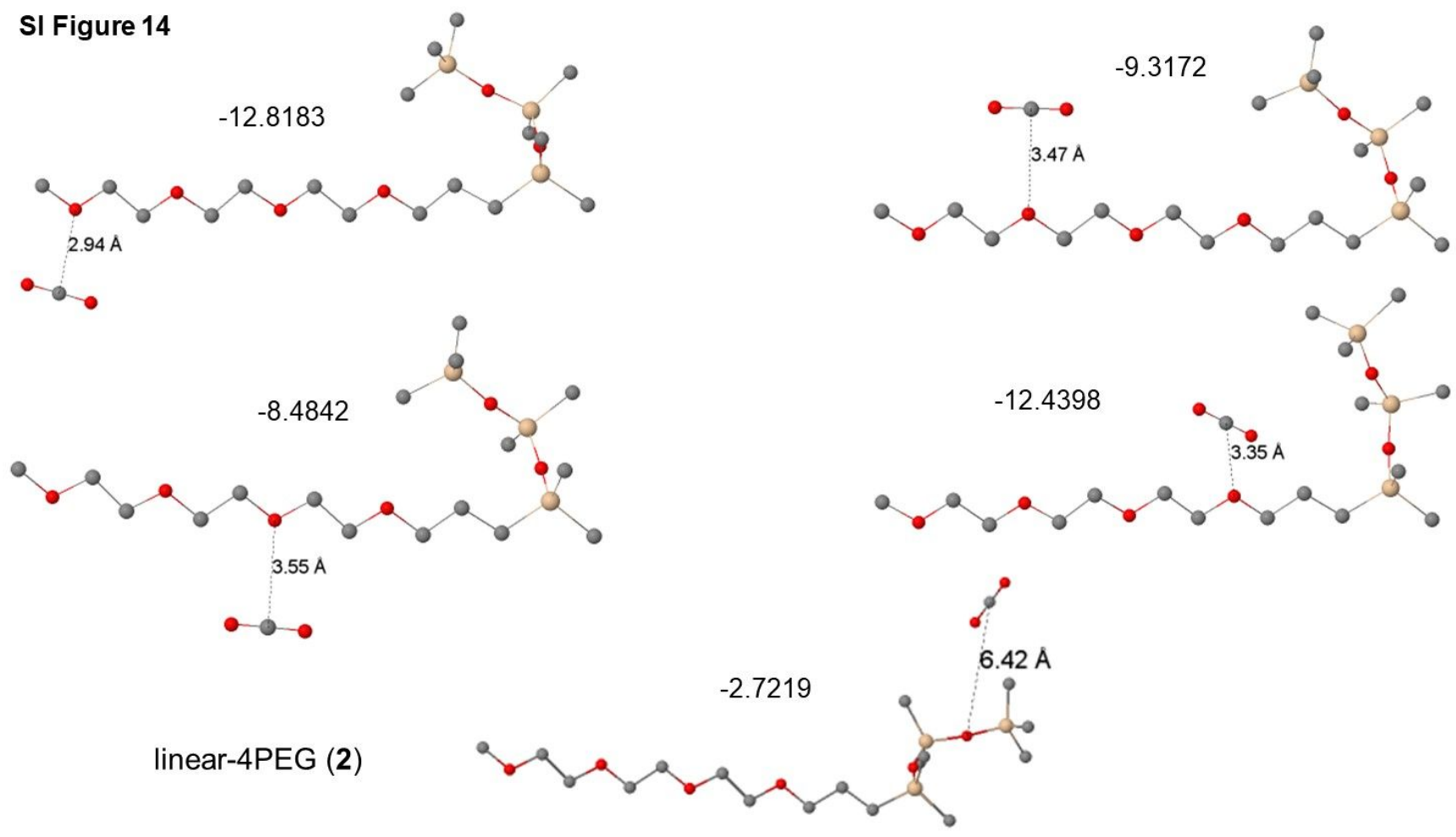

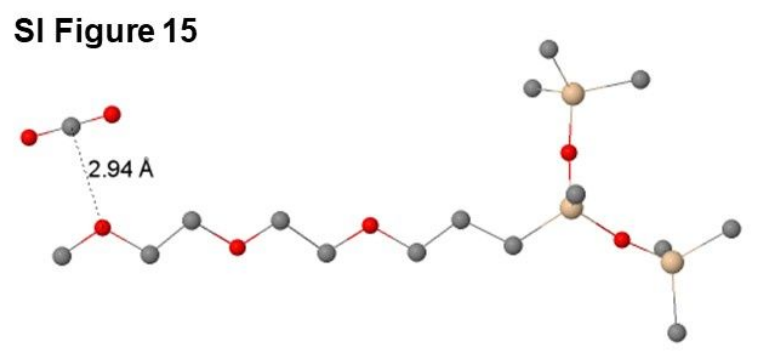

$-12.8140$

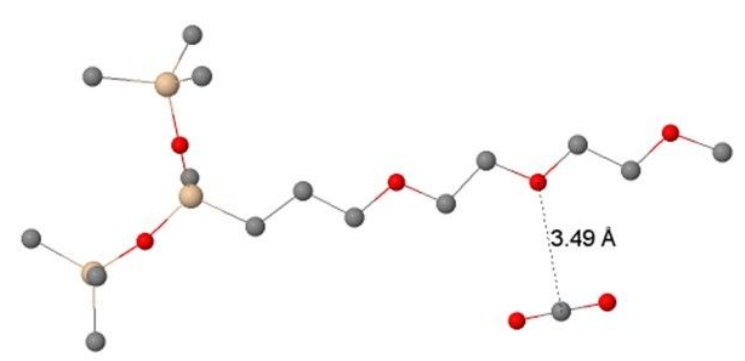

$-9.2355$

T-3PEG (4)

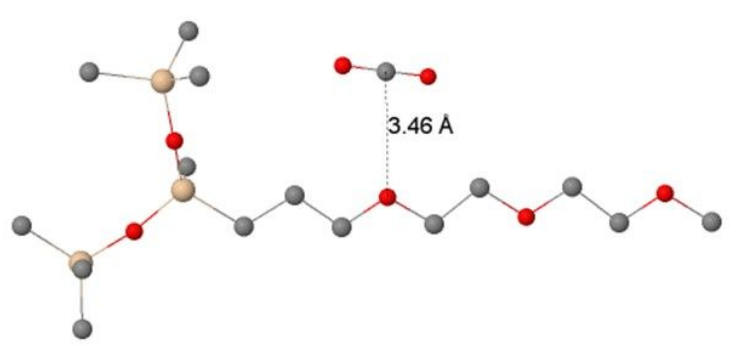

$-11.5709$ 


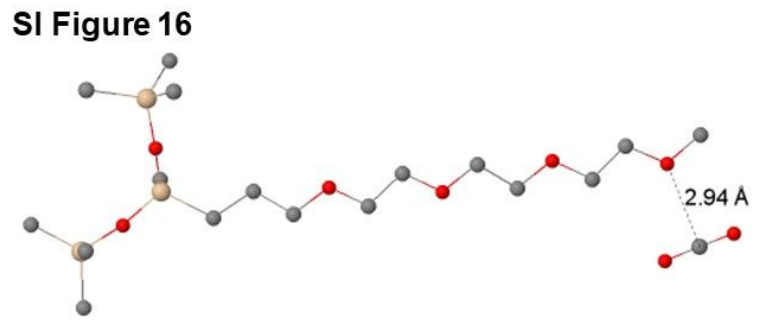

$-12.7968$

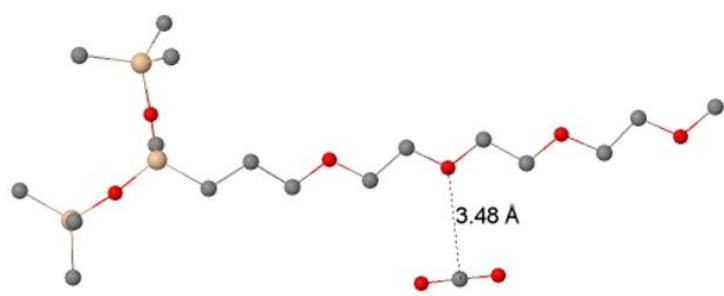

$-9.1752$

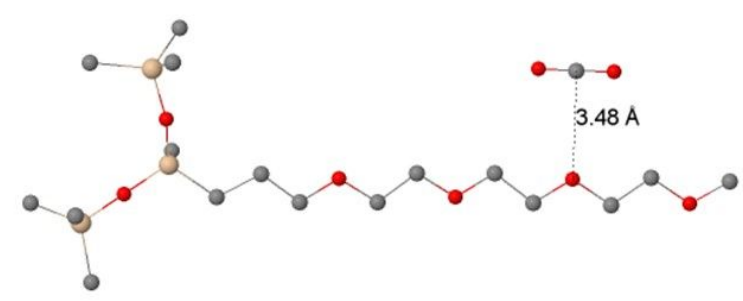

$-9.1784$

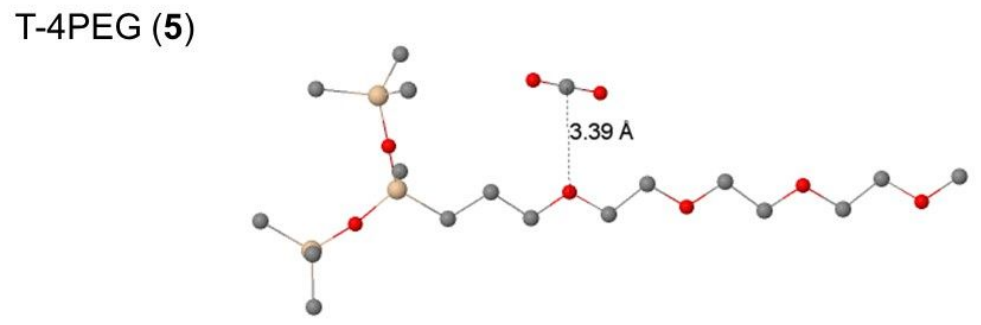

$-11.9828$

SI Figure 17

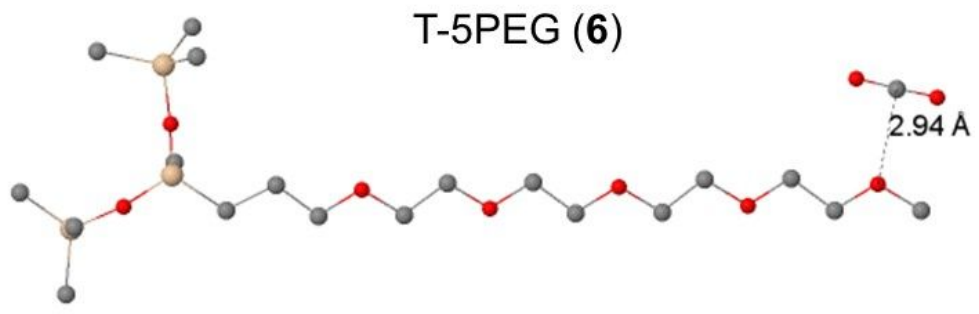

$-12.8088$ 